\title{
FREE ENERGIES IN THE FREQUENCY DOMAIN: THE SCALAR CASE
}

$\mathrm{BY}$

\author{
J. M. GOLDEN
}

School of Mathematics and Computing, Dublin Institute of Technology, Kevin Street, Dublin 8, Ireland

Abstract. A general closed expression is given for the isothermal minimum free energy of a linear viscoelastic material in terms of Fourier-transformed quantities. A oneparameter family of free energies is constructed, ranging continuously from the maximum to the minimum free energies.

The simplest case of single component stress and strain tensors and a single viscoelastic function is considered in this paper. Explicit formulae are given for the particular case of a discrete spectrum model material response.

1. Introduction. We address the problem of finding general, explicit forms for the free energy of a viscoelastic solid. The investigation of this issue has a long history.

Early work on the determination of the free energy of a linear viscoelastic solid by Staverman and Schwarzl [1] involved arguments based on mechanical models. These authors perceived the problem of non-uniqueness and used detailed model assumptions to deal with the issue. Their results were given independently by others (for example $[2,3])$.

Breuer and Onat [4] consider the question: what is the maximum amount of work recoverable from a body that has undergone a specified strain history? They find that the answer is in general provided by the solution of an integral equation of the Wiener-Hopf type, but give a detailed solution by elementary means for a material with relaxation function in the form of a finite sum of decaying exponentials. This response, with or without a constant term (there was no constant in the Breuer-Onat relaxation function) will be referred to below as a discrete spectrum model. The resulting quantity was found to be greater than or equal to the free energy given in $[1,2,3]$. The non-uniqueness problem was also explicitly exposed by Breuer and Onat [5] by first proposing a general form for the free energy of a linear viscoelastic non-aging solid as a quadratic functional

Received March 25, 1998.

2000 Mathematics Subject Classification. Primary 74A15,74D05; Secondary 30E20.

My thanks to M. Fabrizio and G. Gentili for several useful conversations, particularly regarding the question of uniqueness of the factorization.

E-mail address: jmgolden@maths1.kst.dit.ie 
of the strain history and then imposing isothermal thermodynamic constraints upon it, which are investigated for discrete spectrum models.

In the general nonlinear, non-isothermal theory of Coleman [6] and Coleman and Mizel [7], a free energy (and other) functionals are assumed to have certain differentiability properties for histories within a specified normed function space. The constitutive equations and a dissipation inequality then follow from the second law of thermodynamics. Certain properties of the free energy functional derived within this general context were used by various later authors to characterise a free energy. These are stated in Sec. 3 and used in this paper.

Coleman and Owen $[8,9]$ present a general, axiomatic formulation of thermodynamics, in which the existence of the free energy and entropy are deduced from more fundamental considerations rather than assumed a priori. They show that for an elastic-plastic material element, there are an infinity of free energies.

Day [10] presents an alternative formulation of the thermodynamics of materials with memory and, in particular, revisits the problem considered in [4] within a more rigorous framework. In [11] he introduces the concept of a (time) reversible extension and gives an expression for the maximum recoverable work and the associated free energy in terms of this concept. An expression for the minimum free energy of a standard linear solid is given in [12].

Fabrizio and Morro [13, 14] examine the implications of thermodynamics for the Fourier transform of the relaxation function derivative. They deduce simple necessary and sufficient conditions for the validity of the second law in isothermal linear viscoelasticity. It follows from these considerations that the Fourier sine transform must be negative definite. This approach provides a very simple demonstration of the symmetry and positivity of the instantaneous and equilibrium elasticity tensors and the relationship between them. The positivity properties will be used later.

Graffi and Fabrizio $[15,16]$ consider expressions for the free energy of discrete spectrum models. They define a state in terms of a finite number of functions evaluated at the current time and seek an expression for the free energy as a quadratic form in these quantities. Non-uniqueness of this form is demonstrated. Morro and Vianello [17] establish, within a general framework [12], the existence of a minimum and maximum free energy.

A concise presentation of and references to many earlier results is contained in [18], including references to the original work on the positivity and symmetry of the limiting elasticity tensors.

Frequency domain representations of linear viscoelastic quantities are systematically presented in $[19,20]$. A class of free energies is constructed, with elements labelled by a weight function. Special cases are the maximum free energy and the Graffi-Volterra form [18]. This latter form, for the case of discrete spectrum models, also emerges from the considerations in [15].

The problems that arise in interpreting the "internal dissipation function" in isothermal linear viscoelasticity given that it is not unique and is in fact zero for the maximum free energy, are discussed in $[21,22]$. They also extend the developments of [16] for discrete spectrum models where states are represented as a finite number of functions at 
the current time. It emerges that the most general free energy that can be constructed as a quadratic function of these variables does not include the maximum free energy. For the case of a single exponential, the state may be represented by the current values of stress and strain.

More recent work by Del Piero and Deseri [23, 24] examines related issues in depth. Based on the general formulation of Coleman and Owen $[8,9]$, they argue that a valid free energy must be a function of state and that, in agreement with [21], the current values of stress and strain define a state for a relaxation function consisting of a single exponential function. They show that different histories of strain can give the same current values of stress and strain but different values of certain functions that obey the requirements of a free energy function. They show this for two cases: the maximum free energy and the single-integral form proposed by Gurtin and Hrusa [25]. Thus, according to their line of argument, these may not be valid free energies because they may not be functions of state. They also explore the applicability of their definition for several other standard forms.

These are deep issues which will not be resolved in the present work. We adopt here the traditional approach that a state is defined by the current strain and the strain history. However, some further remarks on this topic are made at the end of Sec. 6 .

While the existence of a minimum free energy has been established $[12,18]$, the only case for which an explicit form has been given is the standard linear model. A more general result is implicit in Breuer and Onat [4]. In this paper, a general closed expression is given for the isothermal minimum free energy of a linear viscoelastic material in terms of Fourier-transformed quantities. A one-parameter family of free energies is constructed, ranging continuously from the maximum to the minimum free energies.

The simplest case of single component stress and strain tensors and a single viscoelastic function is considered. The full tensor problem will be treated in a separate paper.

Detailed, explicit formulae are given for the particular material response of the discrete spectrum model. The result of Day [12] is reproduced and the connection with [4] is made explicit. A more complete exploration of the set of free energies associated with a discrete spectrum material is presented in a forthcoming paper.

The layout of the paper is as follows. In Sec. 2, fundamental relationships are written down and a factorisation result required in Sec. 4 is proved. In Sec. 3, the defining properties of a free energy are given and general quadratic functional forms of free energies, both in the time and frequency domains, are discussed. In Sec. 4, the general expression for the minimum free energy is derived and a family of free energies is constructed. In Sec. 5 , these are shown to be special cases of the general quadratic form in the frequency domain, considered in Sec. 3. Finally, the results for discrete spectrum materials are presented in Sec. 6.

2. Basic relationships. We consider a linear viscoelastic solid, subject to stress in such a way that there is only one nonzero component of stress $T(t)$ and strain $E(t)$ related by

$$
\begin{aligned}
T(t) & =G_{0} E(t)+\int_{0}^{\infty} d s G^{\prime}(s) E^{t}(s), \\
E^{t}(s) & =E(t-s), \quad s \in R,
\end{aligned}
$$


where both $E^{t}$ and $G^{\prime} \in L^{1}\left(R^{+}\right) \cap L^{2}\left(R^{+}\right) \cap C^{1}\left(R^{+}\right)$using the notation here and below: $R$ is the set of reals, $R^{+}$the positive reals, and $R^{++}$the strictly positive reals; similarly $R^{-}, R^{--}$are the negative and strictly negative reals. The relaxation function $G(s)$ may be defined by integrating $G^{\prime}(u)$ from $u=0$ to give $G(s)-G_{0}$, with $G_{0}$ identified as $G(0)$. The quantity $G_{\infty}=\lim _{s \rightarrow \infty} G(s)$ is well-defined and we take it to be positive so that the body is a solid. The quantity $G_{0}$ is positive. This follows, in fact, in the general three-dimensional case, from application of arguments based on thermodynamics [18].

For any $f \in L^{2}(R)$, we denote its Fourier transform by

$$
f_{F}(\omega)=\int_{-\infty}^{\infty} d \xi f(\xi) e^{s-i \omega \xi}, \quad f_{F} \in L^{2}(R) .
$$

If $f$ is a real-valued function-which will be the case for all functions of interest here, in the time domain-then $\bar{f}_{F}(\omega)=f_{F}(-\omega)$ where the bar denotes complex conjugate. Functions on $R$ that vanish identically on $R^{--}$are defined as functions on $R^{+}$. For such quantities, $f_{F}=f_{c}-i f_{s}$ where $f_{c}, f_{s}$ are the Fourier cosine and sine transforms:

$$
\begin{aligned}
& f_{c}(\omega)=\int_{0}^{\infty} d \xi f(\xi) \cos \omega \xi=f_{c}(-\omega), \\
& f_{s}(\omega)=\int_{0}^{\infty} d \xi f(\xi) \sin \omega \xi=-f_{s}(-\omega) .
\end{aligned}
$$

Thus

$$
G_{F}^{\prime}(\omega)=\int_{0}^{\infty} d s G^{\prime}(s) e^{s-i \omega s}=G_{c}^{\prime}(\omega)-i G_{s}^{\prime}(\omega) .
$$

Properties of $G_{s}^{\prime}(\omega)$ include [18]

$$
\begin{aligned}
G_{s}^{\prime}(\omega) & <0, \quad \forall \omega \in R^{++}, \\
G_{s}^{\prime}(-\omega) & =-G_{s}^{\prime}(\omega), \quad \forall \omega \in R,
\end{aligned}
$$

the first relation being a consequence of the second law of thermodynamics and the second being a particular case of (2.3). It follows that $G_{s}^{\prime}(0)=0$. We also have [18]

$$
G_{\infty}-G_{0}=\frac{1}{\pi} \int_{-\infty}^{\infty} d \omega \frac{G_{s}^{\prime}(\omega)}{\omega}<0
$$

so that $G_{s}^{\prime}(\omega) / \omega \in L^{1}(R)$.

Let $\Omega$ be the complex $\omega$-plane and let

$$
\begin{aligned}
\Omega^{+} & =\left\{\omega \in \Omega \mid \Im \omega \in R^{+}\right\}, \\
\Omega^{(+)} & =\left\{\omega \in \Omega \mid \Im \omega \in R^{++}\right\} .
\end{aligned}
$$

These define the upper half-plane including and excluding the real axis. Similarly, $\Omega^{-}$, $\Omega^{(-)}$are the lower half-planes including and excluding the real axis, respectively.

The function $G_{F}^{\prime}(\omega)$ given by (2.4) is analytic on $\Omega^{(-)}$. This is a consequence of the fact that $G^{\prime}$ vanishes on $R^{--}$, which is essentially the requirement of Causality [26]. For a discrete spectrum model (see Sec. 6), the singularity structure of $G_{F}^{\prime}$ consists of isolated poles on the positive imaginary axis, while for a continuous spectrum (where $G(t)$ is an integral over a positive function with a decaying exponential factor), there is a cut along a subset of the positive imaginary axis. 
It is assumed that $G_{F}^{\prime}(\omega)$ is analytic on $R$ and therefore on $\Omega^{-}$. For $\omega \in \Omega^{(+)}$, relation (2.4) cannot in general be used to define $G_{F}^{\prime}(\omega)$. Instead, it is defined by analytic continuation from the region of analyticity, namely $\Omega^{-}$, and possibly a strip of $\Omega^{(+)}$. Analyticity at infinity is assumed.

The quantity $G_{F}^{\prime}(-\omega)=\bar{G}_{F}^{\prime}(\omega)$ is analytic in $\Omega^{+}$, a mirror image, in the real axis, of the structure of $G_{F}^{\prime}(\omega)$. Thus, $G_{s}^{\prime}(\omega)$ has singularities in both $\Omega^{(+)}$and $\Omega^{(-)}$that are mirror images of one another. Similarly, its zeros will be mirror images of one another. We will be interested in the singularity structure of

$$
\begin{aligned}
H(\omega) & =-\omega G_{s}^{\prime}(\omega)=H(-\omega) \geq 0, \quad \omega \in R \\
& =H_{1}\left(\omega^{2}\right) .
\end{aligned}
$$

This latter relation is a consequence of the even signature and analyticity of $H(\omega)$ on the real axis. It follows that $H(\omega)$ goes to zero at least quadratically at the origin. It will be assumed that the behaviour is no stronger than quadratic. This is equivalent to the assumption that $\int_{0}^{\infty} d s G^{\prime}(s) s$ is finite and not zero, as can be seen by considering $\lim _{\omega \rightarrow 0} H(\omega) / \omega^{2}$. It will be required in later developments that $H(\omega)$ can be written in the form

$$
H(\omega)=H_{+}(\omega) H_{-}(\omega)
$$

where $H_{+}(\omega)$ has no singularities and zeros in $\Omega^{(-)}$and is thus analytic in $\Omega^{-}$. Similarly, $H_{-}(\omega)$ is analytic in $\Omega^{+}$with no zeros in $\Omega^{(+)}$. We show that it is always possible to do this, given the assumed structure of $H(\omega)$. The argument is similar to those used in the solution of Hilbert problems [27].

By considering the inverse sine transform of $G_{s}^{\prime}(\omega)$ ([18] for example) one can show that

$$
\begin{aligned}
H_{\infty} & =\lim _{|\omega| \rightarrow \infty} H(\omega) \\
& =-\lim _{\omega \rightarrow \infty} \omega G_{s}^{\prime}(\omega)=-G^{\prime}(0) \geq 0 .
\end{aligned}
$$

The sign of $G^{\prime}(0)$ has been deduced by various authors from thermodynamic constraints in the general three-dimensional case $[28,10,18]$. We assume for present purposes that $G^{\prime}(0)$ is nonzero so that $H_{\infty}$ is a finite, positive number. Then $H(\omega) \in R^{++} \forall \omega \in$ $R, \omega \neq 0$. The function

$$
\begin{aligned}
K(\omega) & =\log [H(\omega) T(\omega)], \\
T(\omega) & =\frac{1}{H_{\infty}}+\frac{1}{\omega^{2}}
\end{aligned}
$$

is a well-defined analytic function on $R$, vanishing as $\omega^{-2}$ for large values of $\omega$. Consider the quantity

$$
\begin{aligned}
& M(z)=\frac{1}{2 \pi i} \int_{-\infty}^{\infty} d \omega \frac{K(\omega)}{\omega-z}, \quad z \in \Omega \backslash R, \\
& M(z) \underset{|z| \rightarrow \infty}{\longrightarrow} 0 .
\end{aligned}
$$


For $z \in \Omega^{(+)}, M(z)=M^{+}(z)$ is a function analytic in $\Omega^{(+)}$, while for $z \in \Omega^{(-)}, M(z)=$ $M^{-}(z)$, which is analytic in $\Omega^{(-)}$. The Plemelj formulae give that

$$
M^{+}(\omega)-M^{-}(\omega)=K(\omega), \quad \omega \in R,
$$

where $M^{ \pm}(\omega)$ are the limiting values of $M^{ \pm}(z)$ as $z$ approaches the real axis from above/below. Then if we put

$$
\begin{aligned}
H_{+}(\omega) & =\frac{\omega h_{\infty}}{\omega-i h_{\infty}} e^{-M^{-}(\omega)}, \\
H_{-}(\omega) & =\frac{\omega h_{\infty}}{\omega+i h_{\infty}} e^{+M^{+}(\omega)} \\
h_{\infty} & =H_{\infty}^{\frac{1}{2}},
\end{aligned}
$$

it follows that $H_{+}(z)$ is analytic and free of zeros in $\Omega^{(-)}$and similarly for $H_{-}(z)$ in $\Omega^{(+)}$. Also (2.9) is obeyed. Noting that $\bar{M}^{ \pm}(\omega)=M^{ \pm}(-\omega)=-M^{\mp}(\omega)$, we see that

$$
\begin{aligned}
H_{ \pm}(\omega) & =H_{\mp}(-\omega)=\bar{H}_{\mp}(\omega), \\
H(\omega) & =\left|H_{ \pm}(\omega)\right|^{2} .
\end{aligned}
$$

There arises the issue of the uniqueness of the factorization, since expressions for free energies developed later depend on the individual factors. Consider two different factorizations and take the ratios of corresponding factors. These ratios can be shown to be analytic everywhere and constant at infinity. Invoking Liouville's theorem, we see that the factorization is unique up to constant multipliers. This residual arbitrariness is eliminated by adopting the specific relations given by (2.14) and obeying (2.15).

Consider now the strain function $E^{t}$. Define

$$
E_{+}^{t}(\omega)=\int_{0}^{\infty} d s E^{t}(s) e^{s-i \omega s}, \quad E_{+}^{t} \in L^{2}(R) .
$$

It is analytic in $\Omega^{(-)}$, a property which will be assumed to extend to $\Omega^{-}$. It is defined in all or part of $\Omega^{(+)}$by analytic continuation.

We allow the possibility, for later applications, that a finite discontinuity occurs in $E^{t}$ at $s=0$. Also, it will not be assumed that $E^{t}(-\infty)$ is zero. However, defining

$$
\begin{aligned}
E_{1}^{t}(s) & =E^{t}(s)-E_{\infty}, \quad s \in R^{-}, \\
E_{\infty} & =E^{t}(-\infty),
\end{aligned}
$$

we assume that $E_{1}^{t}: R^{-} \rightarrow R \in L^{1}\left(R^{-}\right) \cap L^{2}\left(R^{-}\right) \cap C^{1}\left(R^{-}\right)$. Let

$$
\begin{aligned}
E_{-}^{t}(\omega) & =E_{r}^{t}(\omega)+E_{\infty} \int_{-\infty}^{0} d s e^{s-i \omega s}=E_{r}^{t}(\omega)-\frac{E_{\infty}}{i \omega^{+}}, \\
E_{r}^{t}(\omega) & =\int_{-\infty}^{0} d s E_{1}^{t}(s) e^{s-i \omega s}, \\
\omega^{+} & =\lim _{\alpha \rightarrow 0^{+}}(\omega+i \alpha) .
\end{aligned}
$$

We have assigned a definite meaning to the right-most integral by giving $\omega$ a small positive imaginary part. The limit $\alpha \rightarrow 0$ is to be taken after any integration involving the quantity $\left(\omega^{+}\right)^{-1}$. Under a similar assumption to that for $E_{+}^{t}$, we may conclude that $E_{r}^{t}$ is analytic in $\Omega^{+}$. It therefore follows that $E_{-}^{t}(\omega)$ is analytic in $\Omega^{+}$. The method just 
described of assigning meaning to the Fourier transforms of functions that do not belong to spaces with sufficient integrability properties will be used later to assign meaning to the transforms of constant histories.

We write

$$
\begin{aligned}
E_{F}^{t}(\omega) & =E_{+}^{t}(\omega)+E_{-}^{t}(\omega) \\
& =\int_{-\infty}^{\infty} d s E^{t}(s) e^{s-i \omega s}=\bar{E}_{F}(\omega) e^{-i \omega t}
\end{aligned}
$$

where

$$
E_{F}(\omega)=\int_{-\infty}^{\infty} d s E(s) e^{s-i \omega s} .
$$

Of course, $E(s)$ does not belong to $L^{1}(R) \cap L^{2}(R)$ if $E_{\infty} \neq 0$ with the consequence that $E_{F}(\omega)$ manifests singular behaviour at the origin. Analyticity at infinity is assumed for $E_{ \pm}^{t}(\omega)$. Note that

$$
\begin{aligned}
& \frac{d E_{+}^{t}(\omega)}{d t}=-i \omega E_{+}^{t}(\omega)+E(t), \\
& \frac{d E_{-}^{t}(\omega)}{d t}=-i \omega E_{-}^{t}(\omega)-E\left(t^{+}\right),
\end{aligned}
$$

where we have allowed for the possibility of a discontinuity in $E^{t}(s)$ at $s=0$. The left-hand limit is simply denoted by $E(t)$ for convenience. The second relation of (2.21) holds only if $E^{t}(s)=E(t-s), s \leq 0$. For the problem considered in Sec. 4 , it will emerge that this is not the case.

Note also that $T(t)$, given by (2.1), is independent of $E^{t}(s), s \in R^{--}$. For the remainder of this section, it will be assumed that $E_{\infty}=0$.

Plancherel's theorem for the Fourier transform $[29,30]$ gives that for any two realvalued functions $g, h \in L^{2}(R)$ we have

$$
\int_{-\infty}^{\infty} d \xi g(\xi) h(\xi)=\frac{1}{2 \pi} \int_{-\infty}^{\infty} d \omega g_{F}(\omega) \vec{h}_{F}(\omega) .
$$

Therefore, we have that

$$
\int_{0}^{\infty} d \xi G^{\prime}(\xi) E^{t}(\xi)=\frac{1}{2 \pi} \int_{-\infty}^{\infty} d \omega G_{F}^{\prime}(\omega) \bar{E}_{F}^{t}(\omega)
$$

where $E_{F}^{t}$ is given by the complex conjugate of the first equation of (2.1) in which the function $\bar{E}_{-}^{t}(\omega)$ may be arbitrarily chosen. One can see in fact, by virtue of the singularity structure of $G_{F}^{\prime}(\omega)$ and $\bar{E}_{-}^{t}(\omega)=E_{-}^{t}(-\omega)$, that by closing the contour on $\Omega^{(-)}$, the term $G_{F}^{\prime}(\omega) \bar{E}_{-}^{t}(\omega)$, in the right-hand integrand of (2.23) will give a vanishing contribution. For this conclusion to be valid, it is necessary that this product, integrated over the infinite portion of the contour, gives zero. The product must therefore vanish as $\omega^{-\alpha}, \alpha>1$ at large $\omega$. This is true because both $G_{F}^{\prime}(\omega)$ and $\bar{E}_{-}^{t}(\omega) \in L^{2}(R)$.

However, it is convenient to retain $\bar{E}_{-}^{t}(\omega)$ in (2.23). Let us choose $E^{t}(s), s \in R^{--}$such that $E^{t}(s), s \in R$ is an odd function. This is equivalent to choosing $\bar{E}_{-}^{t}(\omega)=-E_{+}^{t}(\omega)$. Then (2.1) becomes

$$
T(t)=G_{0} E(t)-\frac{1}{2 \pi i} \int_{-\infty}^{\infty} d \omega G_{s}^{\prime}(\omega)\left[E_{+}^{t}(\omega)-E_{+}^{t}(-\omega)\right] .
$$


This form is more convenient in the present context than the equivalent real form on $R^{+}$, given in $[19,20]$.

Let

$$
T_{1}(t)=T(t)-G_{0} E(t) .
$$

The derivative of this quantity, which will be required later, can be determined from (2.21) and (2.24) to be

$$
\dot{T}_{1}(t)=-\frac{1}{2 \pi} \int_{-\infty}^{\infty} d \omega H(\omega)\left(E_{+}^{t}(\omega)+E_{+}^{t}(-\omega)\right) .
$$

3. Free energies. Let $\psi(t)$ be a free energy functional for the system under consideration. Then the Clausius-Duhem inequality requires, by virtue of standard arguments $[6,7]$ adapted to the isothermal case, that

$$
T(t)=\frac{\partial \psi(t)}{\partial E(t)}
$$

and

$$
\begin{aligned}
T(t) \dot{E}(t) & =D(t)+\dot{\psi}(t) \\
D(t) & \geq 0 \\
\psi(t) & \geq 0
\end{aligned}
$$

provided that $\psi(t)$ has certain differentiability properties. The quantity $D(t)$ is the internal dissipation function. It must be nonnegative as a consequence of the second law. Certain other properties of $\psi(t)$ can be proved within a general framework.

An alternative approach, in the isothermal case ([18] for example), is simply to define a free energy as a functional obeying certain conditions. These express the content of (3.2) and include the other properties referred to above.

The functional $\psi(t)$ is a free energy for the system under consideration if it obeys (3.1) and the following conditions:

C1: It is equal to the elastic free energy for static histories of strain.

C2: For any history of strain, it is not less than the elastic free energy.

C3: The work done by the straining process over any time interval dominates the change in $\psi(t)$ over that interval-the integrated dissipation inequality [6]. The positivity of $\psi(t)$ follows from $\mathrm{C} 2$ since the elastic free energy is conventionally taken to be positive. The first two relations of (3.2) and C3 are essentially equivalent if $\psi(t)$ is differentiable. The key requirement is that $D(t)$ is nonnegative.

We will follow the second approach, but will explore the validity of C3 by deciding whether $D(t)$ is nonnegative or not.

The most general quadratic form for $\psi(t)$, corresponding to a linear non-aging material and a differentiable history, is the well-known functional [5]

$$
\begin{aligned}
\psi(t) & =\frac{1}{2} \int_{0}^{\infty} d s_{1} \int_{0}^{\infty} d s_{2} \dot{E}^{t}\left(s_{1}\right) G\left(s_{1}, s_{2}\right) \dot{E}^{t}\left(s_{2}\right), \\
\dot{E}^{t}(s) & =\frac{d E^{t}(s)}{d s}
\end{aligned}
$$

where $E^{t}(s)$ is defined by (2.1). Also, $G \in C^{3}\left(R^{+} \times R^{+}\right)$. This function and its derivatives 
are assumed to have integrability properties on $R^{+} \times R^{+}$as required. It can be taken to be symmetric in its arguments. The functional $\psi(t)$ obeys the first relation of (3.2) if

$$
\begin{aligned}
D(t) & =-\frac{1}{2} \int_{0}^{\infty} d s_{1} \int_{0}^{\infty} d s_{2} \dot{E}^{t}\left(s_{1}\right) K\left(s_{1}, s_{2}\right) \dot{E}^{t}\left(s_{2}\right) \\
K\left(s_{1}, s_{2}\right) & =G_{1}\left(s_{1}, s_{2}\right)+G_{2}\left(s_{1}, s_{2}\right) \\
G(0, s) & =G(s, 0)=G(s)
\end{aligned}
$$

where subscripts 1,2 indicate differentiation with respect to the first and second argument, respectively. Both $\psi(t)$ and $D(t)$ must be nonnegative for all nonzero histories so that $G\left(s_{1}, s_{2}\right)$ and $-K\left(s_{1}, s_{2}\right)$ must be positive operators.

It will be perceived that there is a potential arbitrariness in the choice of $G\left(s_{1}, s_{2}\right)$. If it is replaced by

$$
\begin{aligned}
G_{n}\left(s_{1}, s_{2}\right) & =G\left(s_{1}, s_{2}\right)+\Gamma\left(s_{1}, s_{2}\right), \\
\Gamma(0, s) & =\Gamma(s, 0)=0
\end{aligned}
$$

where $G_{n}$ and the negative sum of its first derivatives retain the positivity property, then $\psi(t)$ and $D(t)$ still obey (3.2). This points to the well-known non-uniqueness of the free energy for materials with memory. One of the aims of the present work is to explore this non-uniqueness.

We can write $(3.3),(3.4)$ in the alternative forms that do not require differentiability of $E^{t}$ :

$$
\begin{gathered}
\psi(t)=\phi(t)+\frac{1}{2} \int_{0}^{\infty} d s_{1} \int_{0}^{\infty} d s_{2}\left[E^{t}\left(s_{1}\right)-E(t)\right] G_{12}\left(s_{1}, s_{2}\right)\left[E^{t}\left(s_{2}\right)-E(t)\right], \\
\phi(t)=\frac{1}{2} G_{\infty} E^{2}(t)
\end{gathered}
$$

provided that

$$
\begin{aligned}
G_{\infty} & =G(s, \infty)=G(\infty, s)=G(\infty), \\
G_{1}(s, \infty) & =G_{2}(\infty, s)=0
\end{aligned}
$$

so that $G_{\infty}$ is the same quantity as defined after (2.1). The quantity $\phi(t)$ is the elastic free energy. Also,

$$
D(t)=-\frac{1}{2} \int_{0}^{\infty} d s_{1} \int_{0}^{\infty} d s_{2}\left[E^{t}\left(s_{1}\right)-E(t)\right] K_{12}\left(s_{1}, s_{2}\right)\left[E^{t}\left(s_{2}\right)-E(t)\right] .
$$

Condition C1 is clearly satisfied for (3.6). Condition C2 requires that $G_{12}\left(s_{1}, s_{2}\right)$ must be a positive operator. Since $D(t)$ is nonnegative, it also follows that $-K_{12}\left(s_{1}, s_{2}\right)$ must be a positive operator.

It is of interest to write (3.6) in the form

$$
\begin{aligned}
& \psi(t)=S(t)+\frac{1}{2} \int_{0}^{\infty} d s_{1} \int_{0}^{\infty} d s_{2} E^{t}\left(s_{1}\right) G_{12}\left(s_{1}, s_{2}\right) E^{t}\left(s_{2}\right), \\
& S(t)=T(t) E(t)-\frac{1}{2} G_{0} E^{2}(t),
\end{aligned}
$$

and (3.8) as 


$$
\begin{aligned}
D(t) & =-U(t)-\frac{1}{2} \int_{0}^{\infty} d s_{1} \int_{0}^{\infty} d s_{2} E^{t}\left(s_{1}\right) K_{12}\left(s_{1}, s_{2}\right) E^{t}\left(s_{2}\right), \\
U(t) & =T_{d}(t) E(t)-\frac{1}{2} K_{0} E^{2}(t)=\left[\dot{T}_{1}(t)+\int_{0}^{\infty} d s G_{12}(0, s) E^{t}(s)\right] E(t), \\
T_{d}(t) & =K_{0} E(t)+\int_{0}^{\infty} d s K^{\prime}(s) E^{t}(s) \\
& =\dot{T}_{1}(t)+G^{\prime}(0) E(t)+\int_{0}^{\infty} d s G_{12}(0, s) E^{t}(s), \\
K(s) & =K(0, s)=K(s, 0) ; K(\infty, s)=K(s, \infty)=0 ; K_{0}=K(0)=2 G^{\prime}(0),
\end{aligned}
$$

where $T_{1}(t)$ is defined by (2.25). We shall now seek to express these relations in the frequency domain. Let $G_{12}: R^{+} \times R^{+} \rightarrow R \in L^{1}\left(R^{+} \times R^{+}\right) \cap L^{2}\left(R^{+} \times R^{+}\right)$. The definition of $G$ is extended to $R$ by

$$
G\left(s_{1}, s_{2}\right)=G\left(\left|s_{1}\right|,\left|s_{2}\right|\right), \quad s_{1}, s_{2} \in R .
$$

Derivatives of this quantity are discontinuous at $s_{1}, s_{2}=0$. Define

$$
N\left(\omega_{1}, \omega_{2}\right)=\int_{-\infty}^{\infty} d s_{1} \int_{-\infty}^{\infty} d s_{2} e^{-i\left(\omega_{1} s_{1}-\omega_{2} s_{2}\right)} G_{12}\left(s_{1}, s_{2}\right) \in L^{2}(R \times R)
$$

and apply Plancherel's theorem to (3.6) and (3.9) to obtain

$$
\begin{aligned}
\psi(t) & =\phi(t)+\frac{1}{8 \pi^{2}} \int_{-\infty}^{\infty} d \omega_{1} \int_{-\infty}^{\infty} d \omega_{2}\left[\bar{E}_{+}^{t}\left(\omega_{1}\right)+\frac{E(t)}{i \omega_{1}^{+}}\right] N\left(\omega_{1}, \omega_{2}\right)\left[E_{+}^{t}\left(\omega_{2}\right)-\frac{E(t)}{i \omega_{2}^{-}}\right] \\
& =S(t)+\frac{1}{8 \pi^{2}} \int_{-\infty}^{\infty} d \omega_{1} \int_{-\infty}^{\infty} d \omega_{2} \bar{E}_{+}^{t}\left(\omega_{1}\right) N\left(\omega_{1}, \omega_{2}\right) E_{+}^{t}\left(\omega_{2}\right)
\end{aligned}
$$

where $\omega^{+}$is defined by (2.18) and $\omega^{-}=\lim _{\alpha \rightarrow 0}(\omega-i \alpha)$. Similarly, from (3.8) and (3.10),

$$
\begin{aligned}
D(t) & =-\frac{1}{8 \pi^{2}} \int_{-\infty}^{\infty} d \omega_{1} \int_{-\infty}^{\infty} d \omega_{2}\left[\bar{E}_{+}^{t}\left(\omega_{1}\right)+\frac{E(t)}{i \omega_{1}^{+}}\right] M\left(\omega_{1}, \omega_{2}\right)\left[E_{+}^{t}\left(\omega_{2}\right)-\frac{E(t)}{i \omega_{2}^{-}}\right] \\
& =-U(t)-\frac{1}{8 \pi^{2}} \int_{-\infty}^{\infty} d \omega_{1} \int_{-\infty}^{\infty} d \omega_{2} \bar{E}_{+}^{t}\left(\omega_{1}\right) M\left(\omega_{1}, \omega_{2}\right) E_{+}^{t}\left(\omega_{2}\right)
\end{aligned}
$$

where

$$
\begin{aligned}
M\left(\omega_{1}, \omega_{2}\right) & =\int_{-\infty}^{\infty} d s_{1} \int_{-\infty}^{\infty} d s_{2} e^{-i\left(\omega_{1} s_{1}-\omega_{2} s_{2}\right)} K_{12}\left(s_{1}, s_{2}\right) \\
& =i\left(\omega_{1}-\omega_{2}\right) N\left(\omega_{1}, \omega_{2}\right)-2\left(P\left(\omega_{1}\right)+\bar{P}\left(\omega_{2}\right)\right) \\
P(\omega) & =\int_{-\infty}^{\infty} d s e^{s-i \omega s} G_{12}\left(s, 0^{+}\right) .
\end{aligned}
$$

One can show that (3.2) is obeyed by differentiating, for example, the second form of (3.13). The second form of $U(t)$ from (3.10) is required and also Fourier's integral theorem at points of discontinuity, giving

$$
\begin{aligned}
\int_{-\infty}^{\infty} d \omega_{1} N\left(\omega_{1}, \omega\right) & =\int_{-\infty}^{\infty} d \omega_{2} N\left(\omega, \omega_{2}\right)=0 \quad \forall \omega \in R \\
\int_{-\infty}^{\infty} d \omega E_{+}^{t}(\omega) & =\pi E(t) .
\end{aligned}
$$


Note that $M\left(\omega_{1}, \omega_{2}\right)$ may not belong to $L^{2}(R \times R)$ and the integral in (3.14) may exist only as a principal value. This is a feature that we will encounter in Sec. 4 also, as a result of time differentiation of integrals over frequency space, because of the worsening of convergence by a factor $\omega$.

It is of interest to ask what is the statement corresponding to (3.5) in frequency space. Using the equivalence of the two forms of (3.13), we see that if $N_{1}\left(\omega_{1}, \omega_{2}\right)$ replaces $N\left(\omega_{1}, \omega_{2}\right)$ where $N_{1}$ and the corresponding $-M_{1}$ are positive operators and where

$$
N_{d}\left(\omega_{1}, \omega_{2}\right)=N_{1}\left(\omega_{1}, \omega_{2}\right)-N\left(\omega_{1}, \omega_{2}\right)
$$

obeys

$$
\int_{-\infty}^{\infty} \frac{d \omega_{1}}{\omega_{1}^{+}} N_{d}\left(\omega_{1}, \omega\right)=\int_{-\infty}^{\infty} \frac{d \omega_{2}}{\omega_{2}^{-}} N_{d}\left(\omega, \omega_{2}\right)=0 \quad \forall \omega \in R,
$$

the new free energy has the desired properties. Strictly, this condition corresponds not to (3.5) but to a similar statement referring to (3.6).

4. A family of free energies. Consider, to begin with, the well-known form $[17,21]$ of the maximum free energy

$$
\psi_{M}(t)=\int_{-\infty}^{t} d t^{\prime} T\left(t^{\prime}\right) \dot{E}\left(t^{\prime}\right)
$$

It follows from (3.2) that, for this choice of $\psi(t)$ the dissipation function $D(t)$ vanishes. We can write $\psi_{M}(t)$ in the form (3.3):

$$
\psi_{M}(t)=\frac{1}{2} \int_{0}^{\infty} d s_{1} \int_{0}^{\infty} d s_{2} \dot{E}^{t}\left(s_{1}\right) G\left(\left|s_{1}-s_{2}\right|\right) \dot{E}^{t}\left(s_{2}\right) .
$$

However, there are difficulties with the forms (3.6) and (3.9) in that $G\left(\left|s_{1}-s_{2}\right|\right)$ is not differentiable at $s_{1}=s_{2}$. In order to derive the form (3.13) in this case, we proceed in a different manner. Applying a partial integration to (4.1) and separating out the instantaneous contribution to the stress, we find that

$$
\begin{aligned}
\psi_{M}(t) & =S(t)-\int_{0}^{\infty} d s \frac{d}{d t} T_{1}^{t}(s) E^{t}(s) \\
& =S(t)-\frac{1}{2 \pi} \int_{-\infty}^{\infty} d \omega \frac{d}{d t} \bar{T}_{1 F}^{t}(\omega) E_{+}^{t}(\omega), \\
T_{1}^{t}(s) & =T_{1}(t-s),
\end{aligned}
$$

on applying Plancherel's theorem and using notation introduced in (2.25). The wellknown form of the Fourier transform of a convolution product gives with the aid of (2.19) that

$$
\bar{T}_{1 F}^{t}(\omega)=G_{F}^{\prime}(\omega) \bar{E}_{F}^{t}(\omega)=G_{F}^{\prime}(\omega) \bar{E}_{+}^{t}(\omega),
$$

where $E_{-}^{t}(\omega)$, which can be chosen arbitrarily, has been put to zero. It follows from (2.26) that

$$
\frac{d}{d t} \bar{T}_{1 F}^{t}(\omega)=i \omega G_{F}^{\prime}(\omega) \bar{E}_{+}^{t}(\omega)+G_{F}^{\prime}(\omega) E(t) .
$$

We now substitute this form back into (4.3). The second term of (4.5) gives a zero contribution because both $G_{F}^{\prime}(\omega)$ and $E_{+}^{t}(\omega)$ are analytic on $\Omega^{(-)}$and the product vanishes on the infinite boundary. Therefore, 


$$
\psi_{M}(t)=S(t)+\frac{1}{2 \pi} \int_{-\infty}^{\infty} d \omega H(\omega)\left|E_{+}^{t}(\omega)\right|^{2}
$$

where the symmetry properties of $G_{c}^{\prime}(\omega), G_{s}^{\prime}(\omega)$ have been used. This corresponds to the second form of (3.13). We can also write it in the form

$$
\psi_{M}(t)=\phi(t)+\frac{1}{2 \pi} \int_{-\infty}^{\infty} d \omega H(\omega)\left|E_{+}^{t}(\omega)-\frac{E(t)}{i \omega^{-}}\right|^{2}
$$

with the aid of (2.6) and (2.24). The single frequency form corresponds to the case where $N\left(\omega_{1}, \omega_{2}\right)$ in (3.13) is a diagonal operator that can be formally represented in terms of a delta-function. In this case, there is no freedom of the kind expressed by (3.17) and (3.18). Relation (4.7) on $R^{+}$has been given in $[19,20]$.

Note that since $H(\omega)$ vanishes quadratically at $\omega=0$, the quantity $\omega^{-}$in (4.7) can be replaced simply by $\omega$.

We now wish to establish an expression for the minimum free energy, and do so by following a procedure analagous to that of Breuer and Onat [4], but in the frequency domain.

We shall use $t$ henceforth in the special sense of the current time at which an expression for the minimum free energy is sought. Consider $\psi_{M}(u), u>t$, given by (4.6). The limit as $u \rightarrow \infty$ gives

$$
\begin{aligned}
\psi_{M}^{t}(\infty) & =\int_{-\infty}^{\infty} d t T(t) \dot{E}(t) \\
& =S(\infty)+\frac{1}{2 \pi} \int_{-\infty}^{\infty} d \omega H(\omega)\left|E_{+}^{t}(\omega)+E_{-}^{t}(\omega)\right|^{2}, \\
S(\infty) & =G_{\infty} E_{\infty}^{2}-\frac{1}{2} G_{0} E_{\infty}^{2}=\phi(\infty)-\frac{1}{2}\left(G_{0}-G_{\infty}\right) E_{\infty}^{2},
\end{aligned}
$$

which may be shown by considering the limit for large $u$ of $E_{+}^{u}(\omega), u>t$. We have allowed that $\psi_{M}^{t}(\infty)$ may depend on $t$. This will be so if there is a discontinuity in the strain $E^{t}(s)$ at $s=0$.

We assume that $E_{+}^{t}(\omega)$ is given and wish to find the choice of $E_{-}^{t}(\omega)$ that maximizes the recoverable work

$$
\begin{aligned}
W_{r} & =S(\infty)-\int_{t}^{\infty} d t^{\prime} T\left(t^{\prime}\right) \dot{E}\left(t^{\prime}\right) \\
& =S(\infty)+\int_{-\infty}^{t} d t^{\prime} T\left(t^{\prime}\right) \dot{E}\left(t^{\prime}\right)-\int_{-\infty}^{\infty} d t^{\prime} T\left(t^{\prime}\right) \dot{E}\left(t^{\prime}\right)=\psi_{M}(t)-\psi^{t}, \\
\psi^{t} & =\psi_{M}^{t}(\infty)-S(\infty)=\frac{1}{2 \pi} \int_{-\infty}^{\infty} d \omega H(\omega)\left|E_{+}^{t}(\omega)+E_{-}^{t}(\omega)\right|^{2} .
\end{aligned}
$$

The term $S(\infty)$ in $W_{r}$ will be justified later. Now, $\psi_{M}(t)$ is given, and so we seek the choice of $E_{-}^{t}(\omega)$ that minimizes $\psi^{t}$. Let $E_{m}^{t}(\omega)$ be that choice, which implies that if we replace it by $E_{m}^{t}(\omega)+k(\omega)$ where $k(\omega)$ is arbitrary, apart from the fact that $\bar{k}(\omega)=k(-\omega)$, that it behaves as $\omega^{-1}$ at large frequencies and that it is analytic in $\Omega^{+}$(any choice of $E_{-}^{t}(\omega)$ must have these properties) and the resulting integral must not be smaller. It is easy to show that this is assured if

$$
\int_{-\infty}^{\infty} d \omega H(\omega) \Re\left[k(-\omega)\left(E_{+}^{t}(\omega)+E_{m}^{t}(\omega)\right)\right]=0 .
$$

Let us impose the equivalent condition that 


$$
\begin{aligned}
\int_{-\infty}^{\infty} d \omega H(\omega) k(-\omega)\left[E_{+}^{t}(\omega)+E_{m}^{t}(\omega)\right]=0 \\
=\int_{-\infty}^{\infty} d \omega H_{+}(\omega) k(-\omega)\left[H_{-}(\omega) E_{+}^{t}(\omega)+H_{-}(\omega) E_{m}^{t}(\omega)\right] .
\end{aligned}
$$

Note that $k(-\omega), H_{+}(\omega)$, and $E_{+}^{t}(\omega)$ are analytic in $\Omega^{-}$while $H_{-}(\omega)$ and $E_{m}^{t}(\omega)$ are analytic in $\Omega^{+}$. Let

$$
\begin{aligned}
Q^{t}(\omega) & =H_{-}(\omega) E_{+}^{t}(\omega) \\
& =q_{-}^{t}(\omega)-q_{+}^{t}(\omega),
\end{aligned}
$$

where

$$
q^{t}(z)=\frac{1}{2 \pi i} \int_{-\infty}^{\infty} d \omega \frac{Q^{t}(\omega)}{\omega-z}
$$

and $q_{-}^{t}(\omega)$ is the limit of $q^{t}(z)$ on the real axis from above. It is analytic in $\Omega^{(+)}$. Also, $q_{+}^{t}(\omega)$ is the limit from below and is analytic in $\Omega^{(-)}$. This inverted notational convention is adopted to retain conformity with other notation introduced earlier. The function $Q^{t}(\omega)$ is analytic on the real axis. Closing the contour in (4.13) on the half-plane not containing $z$, we pick up the singularities of $Q^{t}(\omega)$ in that half-plane and see that $q_{ \pm}^{t}$ are both analytic on $R$. We have $\bar{q}_{ \pm}^{t}(\omega)=q_{ \pm}^{t}(-\omega), \omega \in R$. The definitions of these functions are extended to $\Omega$ by analytic continuation.

The product $H_{+}(\omega) k(-\omega) q_{+}^{t}(\omega)$ vanishes as $\omega^{-2}$ for large $\omega$ since $k(-\omega)$ and $q_{+}^{t}(\omega)$ vanish as $\omega^{-1}$. Therefore, the integral of this function over the real axis can be extended to an infinite contour on $\Omega^{(-)}$without altering its value. The result is zero because of the analyticity of the integrand on $\Omega^{(-)}$. Therefore, (4.11) becomes

$$
\int_{-\infty}^{\infty} d \omega H_{+}(\omega) k(-\omega)\left[q_{-}^{t}(\omega)+H_{-}(\omega) E_{m}^{t}(\omega)\right]=0 .
$$

This will be true for arbitrary $k(-\omega)$ only if the expression in brackets is a function that is analytic in $\Omega^{(-)}$. However, $E_{m}^{t}(\omega)$ must be analytic in $\Omega^{+}$. Remembering that $q_{-}^{t}(\omega)$ and $H_{-}(\omega)$ are also analytic in $\Omega^{+}$, we see that the expression in brackets must be analyic in both the upper and lower half-planes and on the real axis. Thus, it is analytic over the entire complex plane. Now $q_{-}^{t}(\omega)$ clearly vanishes as $\omega^{-1}$ at infinity, as also must $E_{m}^{t}(\omega)$ if the strain function is to be finite at $s=0$. Therefore, the function is analytic everywhere, zero at infinity, and consequently must vanish everywhere by Liouville's theorem. Thus,

$$
\begin{aligned}
E_{m}^{t}(\omega) & =-\frac{q_{-}^{t}(\omega)}{H_{-}(\omega)} \\
& =-\frac{1}{2 \pi i} \frac{1}{H_{-}(\omega)} \int_{-\infty}^{\infty} d \omega^{\prime} \frac{H_{-}\left(\omega^{\prime}\right) E_{+}^{t}\left(\omega^{\prime}\right)}{\omega^{\prime}-\omega^{+}} .
\end{aligned}
$$

Observe that the pole at the origin due to $H_{-}(\omega)$ in the denominator must be shifted to $\Omega^{(-)}$, i.e., $\left[H_{-}(\omega)\right]^{-1}$ behaves as $\left(\omega^{+}\right)^{-1}$ near the origin. Closing the contour on $\Omega^{(-)}$, we see that the integral picks out the singularities of $H_{-}(\omega)$. It is shown in Sec. 6 that 
this formula gives results in agreement with Breuer and Onat [4] for the particular type of material response considered by them.

Note that the method used to derive (4.15) has similarities in certain aspects to the method of solution of Hilbert problems [27] and to the Wiener-Hopf method that Breuer and Onat [4] and Day [11] refer to. An elementary discussion of this latter technique may be found in [31].

Substituting (4.15) into (4.9), we see that the minimum value of $\psi^{t}$ is given by

$$
\psi_{m}^{t}=\frac{1}{2 \pi} \int_{-\infty}^{\infty} d \omega\left|q_{+}^{t}(\omega)\right|^{2} .
$$

Also, from (4.6)

$$
\begin{aligned}
\psi_{M I}(t) & =S(t)+\frac{1}{2 \pi} \int_{-\infty}^{\infty} d \omega\left|q_{+}^{t}(\omega)-q_{-}^{t}(\omega)\right|^{2} \\
& =S(t)+\frac{1}{2 \pi} \int_{-\infty}^{\infty} d \omega\left[\left|q_{+}^{t}(\omega)\right|^{2}+\left|q_{-}^{t}(\omega)\right|^{2}\right]
\end{aligned}
$$

since the cross-term

$$
q_{-}^{t}(-\omega) q_{+}^{t}(\omega)+q_{-}^{t}(\omega) q_{+}^{t}(-\omega)
$$

consists of terms that are analytic in $\Omega^{(-)}$and $\Omega^{(+)}$, respectively, and that vanish as $\omega^{-2}$ on the infinite boundary. By closing the contour on the half-plane over which a given term is analytic, one obtains zero.

It is clear on physical grounds that any recovered work is a lower bound on any free energy. Thus, if the maximum recoverable work has the properties of a free energy, it must be the minimum free energy. We will make this identification and show that the desired properties hold.

The minimum free energy $\psi_{m}(t)$ is put equal to the quantity $W_{r}$ evaluated for $\psi^{t}=\psi_{m}^{t}$, giving

$$
\psi_{m}(t)=S(t)+\frac{1}{2 \pi} \int_{-\infty}^{\infty} d \omega\left|q_{-}^{t}(\omega)\right|^{2} \leq \psi_{M}(t)
$$

We can write $(4.18)$ in alternative forms:

$$
\begin{aligned}
\psi_{m}(t) & =S(t)+\frac{1}{2 \pi} \int_{-\infty}^{\infty} d \omega H(\omega)\left|E_{m}^{t}(\omega)\right|^{2} \\
& =\phi(t)+\frac{1}{2 \pi} \int_{-\infty}^{\infty} d \omega H(\omega)\left|E_{m}^{t}(\omega)+\frac{E(t)}{i \omega^{+}}\right|^{2} .
\end{aligned}
$$

The latter form follows by the same manipulation as was used to relate (4.7) to (4.6), and is true provided that

$$
\int_{-\infty}^{\infty} d \omega G_{s}^{\prime}(\omega) \Im\left[E_{+}^{t}(\omega)+E_{m}^{t}(\omega)\right]=0 .
$$

This condition is a special case of (4.10) obtained by taking $k(-\omega)=\left(i w^{-}\right)^{-1}$. The restriction to the imaginary part may be removed by symmetry. The resulting relation may be proved directly with the aid of (4.12) and (4.15), by showing that the integrand has the form $H_{+}(\omega) q_{+}^{t}(\omega) / \omega$. It is interesting to connect (4.20) with the result in [11] identifying the history associated with maximum energy recovery with the concept of a reversible extension, which gives a condition similar to the integral equation derived in 
[4]. Equation (4.20) is equivalent to this condition at time $t$ (taken to be zero in [11]), as may be established with the aid of $(2.24)$.

Relation (3.1) is clearly obeyed by $\psi_{m}(t)$ given by the first form of (4.19). Also, condition C2 is manifestly satisfied by the second form of (4.19).

For a static history, $E_{+}^{t}(\omega)$ is given by $E(t) /\left(i \omega^{-}\right)$. Substituting this form into the second relation of (4.15) and closing the contour over $\Omega^{(+)}$, we obtain for a static history

$$
E_{m}^{t}(\omega)=-\frac{E(t)}{i \omega^{+}}
$$

so that the integral in the second form of (4.19) vanishes. Thus, the condition $\mathrm{C} 1$ is obeyed. Note that C1 implies that the maximum recoverable energy from a constant history $E^{t}=E(\infty)$ is $\phi(\infty)$. From a physical standpoint, it is clearly an upper bound of the recoverable energy, which can be approached as closely as desired by releasing the stored energy sufficiently slowly. It justifies the inclusion of $S(\infty)$ in (4.9).

The method of defining the maximum recoverable energy adopted here differs from that of Day [11, 12] (see also [18]). In particular, $\phi(t)$ is not added to it to form $\psi_{m}(t)$.

In order to verify $\mathrm{C} 3$, we must show that

$$
D_{m}(t)=T(t) \dot{E}(t)-\dot{\psi}_{m}(t)
$$

is positive. From the third relation of (4.9) and (4.16) we see that

$$
D_{m}(t)=\frac{d}{d t} \psi_{m}^{t}=\frac{1}{2 \pi} \frac{d}{d t} \int_{-\infty}^{\infty} d \omega\left|q_{+}^{t}(\omega)\right|^{2} .
$$

Note that the first form of (4.9) appears to give a different result. This is because, as we shall see below, there is a discontinuity in the history at time $t$. Before proceeding further, we derive certain auxiliary results, which will be required for this and other purposes. Using (2.21) in (4.13), we find that

$$
\begin{aligned}
\frac{d q_{ \pm}^{t}(\omega)}{d t} & =-i \omega q_{ \pm}^{t}(\omega)-K_{0}(t)+\frac{E(t)}{2 \pi i} \int_{-\infty}^{\infty} d \omega^{\prime} \frac{H_{-}\left(\omega^{\prime}\right)}{\omega^{\prime}-\omega^{\mp}} \\
K_{0}(t) & =\frac{1}{2 \pi} \int_{-\infty}^{\infty} d \omega H_{-}(\omega) E_{+}^{t}(\omega)=\bar{K}_{0}(t),
\end{aligned}
$$

so that

$$
\begin{aligned}
\frac{d}{d t} q_{+}^{t}(\omega) & =-i \omega q_{+}^{t}(\omega)-K(t), \\
\frac{d}{d t} q_{-}^{t}(\omega) & =-i \omega q_{-}^{t}(\omega)-K(t)+H_{-}(\omega) E(t), \\
K(t) & =K_{0}(t)+\frac{1}{2} h_{\infty} E(t) \\
& =\frac{1}{2 \pi} \int_{-\infty}^{\infty} d \omega H_{-}(\omega)\left[E_{+}^{t}(\omega)-\frac{E(t)}{i \omega^{-}}\right] .
\end{aligned}
$$

These results follow from contour integration of the integral in the first relation of (4.24) over $\Omega^{(+)}$. This integral and $K_{0}(t)$ exist in the sense of limits or principal values. They are conveniently evaluated by closing the contour, remembering that there is a contribution from the infinite portion of the contour. The comment after (3.16) will be recalled in this connection. For a static history, $K(t)$ vanishes.

We also require the integrals of $q_{+}^{t}(\omega)$ and $q_{-}^{t}(\omega)$ over the real axis. These can once again be evaluated using the analyticity of the functions over $\Omega^{-}$and $\Omega^{+}$, respectively, 
and the technique mentioned in the previous paragraph. The forms of $q_{ \pm}^{t}(\omega)$ at large $\omega$ must be evaluated with care. We write

$$
\begin{aligned}
q_{ \pm}^{t}(\omega)= & \frac{1}{2 \pi i} \int_{-\infty}^{\infty} d \omega^{\prime} \frac{H\left(w^{\prime}\right)\left[E_{+}^{t}\left(w^{\prime}\right)-\frac{E(t)}{i w^{\prime}}\right]}{w^{\prime}-w^{\mp}} \\
& +\frac{E(t)}{2 \pi i} \int_{-\infty}^{\infty} d \omega^{\prime} \frac{H_{-}\left(\omega^{\prime}\right)}{\left(\omega^{\prime}-\omega^{\mp}\right) i \omega^{\prime-}} .
\end{aligned}
$$

The last integral vanishes for $q_{+}^{t}(\omega)$ and gives $E(t) H_{-}(\omega) /(i \omega)$ for $q_{-}^{t}(\omega)$. Thus

$$
\begin{aligned}
& \lim _{|\omega| \rightarrow \infty} \omega q_{+}^{t}(\omega)=i K(t), \\
& \lim _{|\omega| \rightarrow \infty} \omega q_{-}^{t}(\omega)=i\left(K(t)-h_{\infty} E(t)\right),
\end{aligned}
$$

and

$$
\begin{aligned}
& \frac{1}{2 \pi} \int_{-\infty}^{\infty} d \omega q_{+}^{t}(-\omega)=-\frac{1}{2} K(t)=\frac{1}{2 \pi} \int_{-\infty}^{\infty} d \omega q_{+}^{t}(\omega), \\
& \frac{1}{2 \pi} \int_{-\infty}^{\infty} d \omega q_{-}^{t}(-\omega)=\frac{1}{2}\left(K(t)-h_{\infty} E(t)\right)=\frac{1}{2 \pi} \int_{-\infty}^{\infty} d \omega q_{-}^{t}(\omega) .
\end{aligned}
$$

The complex conjugates of relations (4.25) are easily established and one deduces from (4.23) that

$$
D_{m}(t)=K^{2}(t),
$$

which is clearly positive so that C3 is satisfied by $\psi_{m}(t)$.

Relation (4.29) can also be deduced from the second form of (4.19) with the aid of (2.6), (4.20), and (4.28); and from the first form of (4.19) using (2.26) and the relation

$$
\int_{-\infty}^{\infty} d \omega H(\omega)\left[E_{+}^{t}(\omega)+E_{m}^{t}(\omega)\right]=-\int_{-\infty}^{\infty} d \omega H_{+}(\omega) q_{+}^{t}(\omega)=\pi h_{\infty} K(t),
$$

which is related to the derivative of (4.20) (without the restriction to the imaginary part). The derivative of (4.20) can be shown to vanish without any convergence difficulties arising, by using the fact that $\frac{d}{d t} q_{+}^{t}(\omega)$ (and $\frac{d}{d t} q_{-}^{t}(\omega)$ ) go to zero as $\omega^{-1}$ at large frequencies. This follows from (4.25) and (4.27). It also allows a direct demonstration of the fact that the time derivative of the cross-terms after (4.17) gives a vanishing contribution, when integrated over $R$. This means that we can use the second form of (4.17) with confidence, for purposes of differentiation.

From (4.15) and (4.27), it follows that

$$
E_{m}^{t}(\omega) \underset{\omega \rightarrow \infty}{\longrightarrow}-\frac{E(t)-K(t) / h_{\infty}}{i \omega^{+}} .
$$

Let us denote the optimal deformation as $E_{o}^{t}(s)$, where

$$
E_{m}^{t}(\omega)=\int_{-\infty}^{0} d s E_{o}^{t}(s) e^{s-i \omega s}
$$

The quantity $E_{o}^{t}(s)$ cannot be written in the form $E_{o}(t-s)$ as may be deduced by comparing (2.21) and the derivative of $E_{m}^{t}(\omega)$ as deduced from (4.15) and (4.25). We can determine the form of $E_{o}^{t}(s), s \in R^{--}$from $E_{m}^{t}(\omega)$ by the formula

$$
E_{o}^{t}(s)=\frac{1}{2 \pi} \int_{-\infty}^{\infty} d \omega E_{m}^{t}(\omega) e^{i w s}, \quad s \in R^{-},
$$


evaluated by closing on $\Omega^{(-)}$. For $s \in R^{+}$, we close on $\Omega^{(+)}$to get zero.

It follows from (4.31) and (4.32) that

$$
\lim _{s \rightarrow 0} E_{o}^{t}(s)=E(t)-K(t) / h_{\infty} .
$$

Thus, as mentioned earlier, the optimal deformation involves a sudden discontinuity at time $t$, of magnitude $K(t) / h_{\infty}[4,10]$.

Also, putting

$$
\kappa(\omega)=\frac{H_{-}(\omega)}{\omega}
$$

we have

$$
E_{m}^{t}(\omega) \underset{\omega \rightarrow 0}{\longrightarrow}-\frac{1}{2 \pi i \omega^{+} \kappa(0)} \int_{-\infty}^{\infty} d \omega^{\prime} \kappa\left(\omega^{\prime}\right) E_{+}^{t}\left(\omega^{\prime}\right)
$$

which gives, on considering (2.18),

$$
E_{o}^{t}(-\infty)=\frac{1}{2 \pi \kappa(0)} \int_{-\infty}^{\infty} d \omega^{\prime} \kappa\left(\omega^{\prime}\right) E_{+}^{t}\left(\omega^{\prime}\right)
$$

This quantity is in general nonzero, in agreement with the observations in [10]. The fact that the optimal deformation does not go to zero at large times is also implicit in the results of [4].

Consider the generalisation of (4.7) and (4.19)

$$
\psi(t)=\phi(t)+\frac{1}{2 \pi} \int_{-\infty}^{\infty} d \omega\left|q_{-}^{t}(\omega)-\alpha(\omega) q_{+}^{t}(\omega)+\frac{i H_{-}(\omega) E(t)}{\omega}\right|^{2}
$$

where $\alpha(\omega)$ is an arbitrary complex function constrained by the requirements that it is analytic in $\Omega^{-}\left(\right.$as is $\left.q_{+}^{t}(\omega)\right)$ and

$$
\left.\begin{array}{rl}
|\alpha(\omega)|^{2} & \leq 1 \\
\alpha(-\omega) & =\bar{\alpha}(\omega)
\end{array}\right\} \omega \in R
$$

Note that $\omega^{ \pm}$has been replaced by $\omega$ since the zero in $H_{-}(\omega)$ cancels this singularity.

Condition C2 is clearly met. Also, for a static history, $q_{+}^{t}(\omega)$ vanishes (see (4.26)) and $q_{-}^{t}(\omega)$ cancels the term proportional to $E(t)$ in (4.31), by virtue of (4.21). Thus, C1 is also obeyed. We can write (4.38) in the form

$$
\psi(t)=S(t)+\frac{1}{2 \pi} \int_{-\infty}^{\infty} d \omega\left[\left|q_{-}^{t}(\omega)\right|^{2}+|\alpha(\omega)|^{2}\left|q_{+}^{t}(\omega)\right|^{2}\right] .
$$

In order to show this, the only new result required is that the cross-term

$$
E(t) \int_{-\infty}^{\infty} d \omega \frac{\alpha(-\omega) q_{+}^{t}(-\omega) H_{-}(\omega)}{\omega}
$$

and its complex conjugate vanish. The integrand is analytic in $\Omega^{+}$and behaves as $\omega^{-2}$ at large $\omega$, so that the result follows. An immediate conclusion from (4.40) is that (3.1) is obeyed.

It remains to consider C3. Differentiating (4.40) with the aid of (4.25) and using (4.28) and (4.29), we find that difficulties arise unless $|\alpha(\omega)|^{2}$ is chosen to be a constant which 
will be denoted by $\alpha^{2}$, where $\alpha$ is real and $|\alpha| \leq 1$. With this restriction, it emerges that

$$
D(t)=\left(1-\alpha^{2}\right) K^{2}(t)
$$

which is positive for $\alpha^{2}<1$.

We have therefore a one-parameter family of free energies connecting $\psi_{M}(t)$ and $\psi_{m}(t)$, given by

$$
\psi_{\alpha}(t)=S(t)+\frac{1}{2 \pi} \int_{-\infty}^{\infty} d \omega\left[\left|q_{-}^{t}(\omega)\right|^{2}+\alpha^{2}\left|q_{+}^{t}(\omega)\right|^{2}\right], \quad 0 \leq \alpha^{2} \leq 1 .
$$

The free energy corresponding to $(-\alpha)$ is equal to that corresponding to $\alpha$. This is apparent from (4.42) though not immediately so from (4.38) with $\alpha(\omega)$ replaced by $\alpha$. Equation (4.42) may be cast as

$$
\psi_{\alpha}(t)=\alpha^{2} \psi_{M}(t)+\left(1-\alpha^{2}\right) \psi_{m}(t)
$$

compatible with the general convexity property of free energy functions [18].

5. Two frequency form. The family (4.42) is expressed as an integral over a single frequency. However, except for the limiting case of the maximum free energy $(\alpha=1)$, it is not diagonal when expressed in terms of the strain histories up to time $t$. In other words, if expressed in the form (3.13), $N\left(\omega_{1}, \omega_{2}\right)$ is not diagonal. It is of interest to write down the form of $N\left(\omega_{1}, \omega_{2}\right)$ for this family. Interchanging integrations, one finds that

$$
N_{\alpha}\left(\omega_{1}, \omega_{2}\right)=\frac{1}{\pi} \int_{-\infty}^{\infty} d \omega\left\{\frac{H_{+}\left(\omega_{1}\right)}{\omega-\omega_{1}^{+}} \frac{H_{-}\left(\omega_{2}\right)}{\omega-\omega_{2}^{-}}+\alpha^{2} \frac{H_{+}\left(\omega_{1}\right)}{\omega-\omega_{1}^{-}} \frac{H_{-}\left(\omega_{2}\right)}{\omega-\omega_{2}^{+}}\right\}
$$

where $\omega_{1}, \omega_{2}$ are moved slightly off the real axis, rather than $\omega$. The $\omega$ integration can be carried out by closing on either half-plane and we obtain

$$
N_{\alpha}\left(\omega_{1}, \omega_{2}\right)=2 i H_{+}\left(\omega_{1}\right) H_{-}\left(\omega_{2}\right)\left\{\frac{1}{\omega_{1}^{+}-\omega_{2}^{-}}-\alpha^{2} \frac{1}{\omega_{1}^{-}-\omega_{2}^{+}}\right\} .
$$

One can show that the second term vanishes under the operations specified in (3.18), as might be expected. Using the Plemelj formulae, we find that

$$
\psi_{\alpha}(t)=S(t)+\frac{\left(1+\alpha^{2}\right)}{4 \pi} \int_{-\infty}^{\infty} d \omega\left|Q^{t}(\omega)\right|^{2}+i \frac{\left(1-\alpha^{2}\right)}{4 \pi^{2}} P \int_{-\infty}^{\infty} d \omega_{1} d \omega_{2} \frac{\bar{Q}^{t}\left(\omega_{1}\right) Q^{t}\left(\omega_{2}\right)}{\omega_{1}-\omega_{2}}
$$

where $Q^{t}(\omega)$ is defined by (4.12) and the " $P$ " in the last term indicates that the first integral evaluated is a principal value. For $\alpha=1$ this form is clearly diagonal. With the aid of (4.6), it can be rewritten as

$$
\begin{aligned}
\psi_{\alpha}(t) & =\psi_{M}(t)+\frac{\left(1-\alpha^{2}\right)}{4 \pi} A \\
A & =-\int_{-\infty}^{\infty} d \omega\left|Q^{t}(\omega)\right|^{2}+\frac{i}{\pi} P \int_{-\infty}^{\infty} d \omega_{1} d \omega_{2} \frac{\bar{Q}^{t}\left(\omega_{1}\right) Q^{t}\left(\omega_{2}\right)}{\omega_{1}-\omega_{2}} \\
& =-2 \int_{-\infty}^{\infty} d \omega\left|q_{+}^{t}(\omega)\right|^{2}
\end{aligned}
$$


6. A particular model. We now consider the results of Sec. 4 for a particular class of response functions, namely discrete spectrum models.

Let the relaxation function $G(t)$ have the form

$$
G(t)=G_{\infty}+\sum_{i=1}^{n} G_{i} e^{-\alpha_{i} t}
$$

where $n$ is a positive integer, the inverse decay times $\alpha_{i} \in R^{+}, i=1,2, \ldots, n$, and the coefficients $G_{i}$ are also generally assumed to be positive. We arrange that $\alpha_{1}<\alpha_{2}<$ $\alpha_{3}<\cdots$. We have

$$
G^{\prime}(t)=\sum_{i=1}^{n} g_{i} e^{-\alpha_{i} t}, \quad g_{i}=-\alpha_{i} G_{i}<0
$$

and

$$
\begin{aligned}
G_{F}^{\prime}(\omega) & =\sum_{i=1}^{n} \frac{g_{i}}{\alpha_{i}+i \omega}, \\
G_{c}^{\prime}(\omega) & =\sum_{i=1}^{n} \frac{\alpha_{i} g_{i}}{\alpha_{i}^{2}+\omega^{2}}, \\
G_{s}^{\prime}(\omega) & =\omega \sum_{i=1}^{n} \frac{g_{i}}{\alpha_{i}^{2}+\omega^{2}},
\end{aligned}
$$

recalling (2.4). Thus

$$
H(\omega)=-\omega^{2} \sum_{i=1}^{n} \frac{g_{i}}{\alpha_{i}^{2}+\omega^{2}} \geq 0
$$

and (2.10) can easily be checked. Observe that $f(z)=H(\omega), z=-\omega^{2}$ has simple poles at $\alpha_{i}^{2}, i=1,2, \ldots, n$. It will therefore have zeros at $\gamma_{i}^{2}, i=2,3, \ldots, n$ where

$$
\alpha_{1}^{2}<\gamma_{2}^{2}<\alpha_{2}^{2}<\gamma_{3}^{2}<\cdots
$$

The function $f(z)$ also vanishes at $\gamma_{1}=0$. Therefore,

$$
H(\omega)=H_{\infty} \prod_{i=1}^{n}\left\{\frac{\gamma_{i}^{2}+\omega^{2}}{\alpha_{i}^{2}+\omega^{2}}\right\}
$$

and either by inspection or by applying the general formula (2.14), one can show that

$$
\begin{aligned}
& H_{+}(\omega)=h_{\infty} \prod_{i=1}^{n}\left\{\frac{\omega-i \gamma_{i}}{\omega-i \alpha_{i}}\right\}, \\
& H_{-}(\omega)=h_{\infty} \prod_{i=1}^{n}\left\{\frac{\omega+i \gamma_{i}}{\omega+i \alpha_{i}}\right\} .
\end{aligned}
$$


We have

$$
\begin{aligned}
H_{-}(\omega) & =h_{\infty}\left[1+i \sum_{i=1}^{n} \frac{R_{i}}{\omega+i \alpha_{i}}\right], \quad H_{+}(\omega)=\bar{H}_{-}(\omega), \\
R_{i} & =\left(\gamma_{i}-\alpha_{i}\right) \prod_{\substack{j=1 \\
j \neq i}}^{n}\left\{\frac{\gamma_{j}-\alpha_{i}}{\alpha_{j}-\alpha_{i}}\right\} .
\end{aligned}
$$

The quantity $q_{-}^{t}(\omega)$ defined by (4.12) may be evaluated by closing on $\Omega^{(-)}$, giving

$$
q_{-}^{t}(\omega)=i h_{\infty} \sum_{i=1}^{n} \frac{R_{i} E_{+}^{t}\left(-i \alpha_{i}\right)}{\omega+i \alpha_{i}}
$$

Also,

$$
\begin{aligned}
q_{+}^{t}(\omega) & =q_{-}^{t}(\omega)-H_{-}(\omega) E_{+}^{t}(\omega) \\
& =i h_{\infty} \sum_{i=1}^{n} \frac{R_{i}\left[E_{+}^{t}\left(-i \alpha_{i}\right)-E_{+}^{t}(\omega)\right]}{\omega+i \alpha_{i}}-h_{\infty} E_{+}^{t}(\omega),
\end{aligned}
$$

which has singularities at those of $E_{+}^{t}(\omega)$ in $\Omega^{(+)}$. From (4.15) and (6.7),

$$
\begin{aligned}
E_{m}^{t}(\omega) & =-i \sum_{i=1}^{n} J_{i}(\omega) R_{i} E_{+}^{t}\left(-i \alpha_{i}\right), \\
J_{i}(\omega) & =\frac{\prod_{\substack{j=1 \\
j \neq i}}^{n}\left(\omega+i \alpha_{j}\right)}{\prod_{j=1}^{n}\left(\omega+i \gamma_{j}\right)}=\sum_{l=1}^{n} \frac{K_{i l}}{\omega+i \gamma_{l}},
\end{aligned}
$$

where

$$
K_{i l}=\frac{\prod_{\substack{j \neq 1 \\ j \neq i}}^{n}\left(\gamma_{l}-\alpha_{j}\right)}{\prod_{\substack{j=1 \\ j \neq l}}^{n}\left(\gamma_{l}-\gamma_{j}\right)}
$$

so that

$$
\begin{aligned}
E_{m}^{t}(\omega) & =-i \sum_{l=1}^{n} \frac{B_{l}^{t}}{\omega+i \gamma_{l}} \\
B_{l}^{t} & =\sum_{i=1}^{n} R_{i} K_{i l} E_{+}^{t}\left(-i \alpha_{i}\right) .
\end{aligned}
$$

We conclude that the optimal deformation as defined in (4.33) has the form

$$
\begin{aligned}
E_{o}^{t}(s) & =-\sum_{l=1}^{n} B_{l}^{t} e^{\gamma_{l} s}, \quad s<0 \\
& =-B_{1}^{t}-\sum_{l=2}^{n} B_{l}^{t} e^{\gamma_{l} s} .
\end{aligned}
$$

Note that

$$
E_{o}^{t}(-\infty)=-B_{1}^{t}
$$

which is a special case of (4.37) 
By considering $\omega J_{i}(\omega)$ for large $\omega$, it can be deduced that

$$
\sum_{l=1}^{n} K_{i l}=1
$$

so that

$$
\begin{aligned}
\sum_{l=1}^{n} B_{l}^{t} & =\sum_{i=1}^{n} R_{i} E_{+}^{t}\left(-i \alpha_{i}\right) \\
& =-E_{o}^{t}(0) .
\end{aligned}
$$

From (4.24), the second to last relation of (4.25), and (6.8) we have

$$
K(t)=h_{\infty}\left[\sum_{i=1}^{n} R_{i} E_{+}^{t}\left(-i \alpha_{i}\right)+E(t)\right]
$$

so that the discontinuity at time $t$ is $K(t) / h_{\infty}$, as determined in the general case. From (6.8) we conclude that

$$
\sum_{i=1}^{n} \frac{R_{i}}{\alpha_{i}}=-1
$$

giving

$$
K(t)=h_{\infty} \sum_{i=1}^{n} \frac{R_{i}}{\alpha_{i}}\left[\alpha_{i} E_{+}^{t}\left(-i \alpha_{i}\right)-E(t)\right],
$$

which also follows from the last relation of (4.25). The quantity $K(t) / h_{\infty}$ given by (6.20) may be related without difficulty to the jump discontinuity term in [4].

Using (4.21) and the static form of $E_{+}^{t}(\omega)$, namely $E(t) /\left(i \omega^{-}\right)$, in (6.13) gives that

$$
\begin{aligned}
& \sum_{i=1}^{n} \frac{R_{i}}{\alpha_{i}} K_{i 1}=-1, \\
& \sum_{i=1}^{n} \frac{R_{i}}{\alpha_{i}} K_{i l}=0, \quad l=2,3, \ldots, n,
\end{aligned}
$$

so that

$$
\begin{aligned}
B_{1}^{t} & =\sum_{i=1}^{n} \frac{R_{i}}{\alpha_{i}} K_{i 1}\left[\alpha_{i} E_{+}^{t}\left(-i \alpha_{i}\right)-E(t)\right]-E(t) \\
B_{l}^{t} & =\sum_{i=1}^{n} \frac{R_{i}}{\alpha_{i}} K_{i l}\left[\alpha_{i} E_{+}^{t}\left(-i \alpha_{i}\right)-E(t)\right]
\end{aligned}
$$

The quantities $\gamma_{l} B_{l}^{t}, l=2,3, \ldots, n$, given by (6.22) can be shown to agree with corresponding quantities in [4]. 
We deduce from (6.9) that

$$
\begin{aligned}
\frac{1}{2 \pi} \int_{-\infty}^{\infty} d \omega\left|q_{-}^{t}(\omega)\right|^{2} & =H_{\infty} \sum_{i, j=1}^{n} \frac{R_{i} R_{j}}{\alpha_{i}+\alpha_{j}} E_{+}^{t}\left(-i \alpha_{i}\right) E_{+}^{t}\left(-i \alpha_{j}\right) \\
& =\frac{1}{2} \int_{0}^{\infty} d s_{1} \int_{0}^{\infty} d s_{2} E^{t}\left(s_{1}\right) F\left(s_{1}, s_{2}\right) E^{t}\left(s_{2}\right) \\
F\left(s_{1}, s_{2}\right) & =2 H_{\infty} \sum_{i, j=1}^{n} \frac{R_{i} R_{j}}{\alpha_{i}+\alpha_{j}} e^{-\alpha_{i} s_{1}-\alpha_{j} s_{2}}
\end{aligned}
$$

and the minimum free energy is given by (4.18). The second relation is cast in the form (3.9), where

$$
F\left(s_{1}, s_{2}\right)=G_{12}\left(s_{1}, s_{2}\right) .
$$

The solution of this partial differential equation compatible with (3.7) is

$$
G\left(s_{1}, s_{2}\right)=G_{\infty}+2 H_{\infty} \sum_{i, j=1}^{n} \frac{R_{i} R_{j}}{\left(\alpha_{i}+\alpha_{j}\right) \alpha_{i} \alpha_{j}} e^{-\alpha_{i} s_{1}-\alpha_{j} s_{2}} .
$$

The last relation of (3.4) can be confirmed with the aid of the identity

$$
\sum_{j=1}^{n} \frac{R_{j}}{\left(\alpha_{i}+\alpha_{j}\right) \alpha_{j}}=-\frac{g_{i}}{2 R_{i} H_{\infty}}
$$

which follows from (6.19) and the identity

$$
\sum_{j=1}^{n} \frac{R_{j}}{\alpha_{i}+\alpha_{j}}=-1+\frac{\alpha_{i} g_{i}}{2 R_{i} H_{\infty}}
$$

which in turn can be deduced by comparing the product $H_{+}(\omega) H_{-}(\omega)$ given by (6.8) near poles of $H_{+}(\omega)$ with $H(\omega)$ given by (6.4).

Comparing (6.4) and (6.6), we deduce that

$$
R_{i} S_{i}=\frac{\alpha_{i}^{2} g_{i}}{H_{\infty}}, \quad i=1,2, \ldots, n
$$

where $R_{i}$ is defined by (6.8) and

$$
S_{i}=\left(\gamma_{i}+\alpha_{i}\right) \prod_{\substack{j=1 \\ j \neq i}}^{n}\left\{\frac{\gamma_{j}+\alpha_{i}}{\alpha_{j}+\alpha_{i}}\right\}
$$

Using (6.28) we can write $G\left(s_{1}, s_{2}\right)$ in the form

$$
G\left(s_{1}, s_{2}\right)=G_{\infty}+\frac{2}{H_{\infty}} \sum_{i, j=1}^{n} \frac{\alpha_{i} \alpha_{j} g_{i} g_{j}}{S_{i} S_{j}\left(\alpha_{i}+\alpha_{j}\right)} e^{-\alpha_{i} s_{1}-\alpha_{j} s_{2}}
$$

which can be seen to be equivalent to the formula of Breuer and Onat [4] (who put $G_{\infty}=0$ ), on perceiving that $S_{i}$ has an extra factor $\alpha_{i}$ on the products used in [4]. 
Noting (3.8), (6.24), and (6.20) we see that (4.29) is obeyed, giving

$$
\begin{aligned}
D_{m}(t) & =H_{\infty}\left\{\sum_{i=1}^{n} \frac{R_{i}}{\alpha_{i}}\left[\alpha_{i} E_{+}^{t}\left(-i \alpha_{i}\right)-E(t)\right]\right\}^{2} \\
& =H_{\infty}\left\{\int_{0}^{\infty} d s \sum_{i=1}^{n} R_{i} e^{-\alpha_{i} s}\left[E^{t}(s)-E(t)\right]\right\}^{2} .
\end{aligned}
$$

Equation (3.2) can be verified directly from (6.23) with the aid of (6.27) and (6.31).

For $n=1$,

$$
\begin{aligned}
\psi_{m}(t) & =S(t)+\frac{1}{2} H_{\infty} \alpha\left\{\int_{0}^{\infty} d s E^{t}(s) e^{-\alpha s}\right\}^{2} \\
& =S(t)+\frac{1}{2} H_{\infty} \alpha\left|E_{+}^{t}(-i \alpha)\right|^{2} \\
& =\phi(t)+\frac{1}{2} H_{\infty} \alpha\left\{\int_{0}^{\infty} d s\left[E^{t}(s)-E(t)\right] e^{-\alpha s}\right\}^{2},
\end{aligned}
$$

which can be shown to agree with the result of Day [12]; see also [18, 24]. Also,

$$
\begin{aligned}
D_{m}(t) & =H_{\infty}\left|\alpha E_{+}^{t}(-i \alpha)-E(t)\right|^{2} \\
& =H_{\infty}\left\{\alpha \int_{0}^{\infty} d s e^{-\alpha s}\left[E^{t}(s)-E(t)\right]\right\}^{2} .
\end{aligned}
$$

In this case, $\psi_{m}(t)$ coincides with the free energy given in $[1,2,3]$ as may be seen from the results of [4]; see also [21]. It can also be shown to be equal to the Graffi-Volterra free energy $[18,15]$.

All of these free energies can be expressed, for general values of $n$, as quadratic forms of a vector consisting essentially of $E(t)$ and $E_{+}^{t}\left(-i \alpha_{i}\right), i=1,2, \ldots, n[21,15]$. This means that they are free energies in the restricted sense of Del Piero and Deseri [23, 24], who in fact prove that for $n=1$ the free energy is unique, a result implying the observations of the previous paragraph. The analysis of $[23,24]$ is for exponential forms of a very general kind.

The maximum free energy $\psi_{M}(t)$ is not a free energy in the restricted sense of $[23,24]$ for discrete spectrum models. This is consistent with (4.17), (6.9), and (6.10), from which we see that $q_{-}^{t}(\omega)$ depends only on $E_{+}^{t}\left(-i \alpha_{i}\right), i=1,2, \ldots, n$ while $q_{+}^{t}(\omega)$ depends also on $E_{+}^{t}(\omega)$. It follows that none of the family of free energies defined at the end of Sec. 4 , except $\psi_{m}(t)$ itself, is a free energy in the restricted sense, for discrete spectrum models, since, from (4.43), they will depend on $\psi_{M}(t)$.

\section{REFERENCES}

[1] A. J. Staverman and F. Schwarzl, Thermodynamics of viscoelastic behaviour, Proc. Konink. Nederl. Akad. Wettensch. B55, 474-485 (1952)

[2] D. Bland, The Theory of Linear Viscoelasticity, Pergamon, London, 1960

[3] S. C. Hunter, Tentative equations for the propagation of stress, strain and temperature fields in viscoelastic solids, J. Mech. Phys. Solids 9, 39-51 (1961)

[4] S. Breuer and E. T. Onat, On recoverable work in linear viscoelasticity, Z. Angew. Math. Phys. 15, 13-21 (1964) 
[5] S. Breuer and E. T. Onat, On the determination of free energy in viscoelastic solids, Z. Angew. Math. Phys. 15, 185-191 (1964)

[6] B. D. Coleman, Thermodynamics of materials with memory, Arch. Rational Mech. Anal. 17, 1-46 (1964)

[7] B. D. Coleman and V. J. Mizel, A general theory of dissipation in materials with memory, Arch. Rational Mech. Anal. 27, 255-274 (1967)

[8] B. D. Coleman and D. R. Owen, A mathematical foundation for thermodynamics, Arch. Rational Mech. Anal. 54, 1-104 (1974)

[9] B. D. Coleman and D. R. Owen, On thermodynamics and elastic-plastic materials, Arch. Rational Mech. Anal. 59, 25-51 (1975)

[10] W. A. Day, Thermodynamics based on a work axiom, Arch. Rational Mech. Anal. 31, 1-34 (1968)

[11] W. A. Day, Reversibility, recoverable work and free energy in linear viscoelasticity, Quart. J. Mech. Appl. Math. 23, 1-15 (1970)

[12] W. A. Day, The thermodynamics of materials with memory, in Materials with Memory, ed. D. Graffi, Liguori, Naples, 1979

[13] M. Fabrizio and A. Morro, Thermodynamic restrictions on relaxation functions in linear viscoelasticity, Mech. Res. Comm. 12, 101-105 (1985)

[14] M. Fabrizio and A. Morro, Viscoelastic relaxation functions compatible with thermodynamics, J. Elasticity 19, 63-75 (1988)

[15] D. Graffi and M. Fabrizio, Sulla nozione di stato materiali viscoelastici di tipo "rate", Atti Accad. Naz. Lincei 83, 201-208 (1990)

[16] D. Graffi and M. Fabrizio, Non unicità dell'energia libera per materiali viscoelastici, Atti Accad. Naz. Lincei 83, 209-214 (1990)

[17] A. Morro and M. Vianello, Minimal and maximal free energy for materials with memory, Boll. Un. Mat. Ital. 4A, 45-55 (1990)

[18] M. Fabrizio and A. Morro, Mathematical Problems in Linear Viscoelasticity, SIAM, Philadelphia, 1992

[19] M. Fabrizio, C. Giorgi, and A. Morro, Free energies and dissipation properties for systems with memory, Arch. Rational Mech. Anal. 125, 341-373 (1994)

[20] M. Fabrizio, Existence and uniqueness results for viscoelastic materials, in Crack and Contact Problems for Viscoelastic Bodies, eds. G. A. C. Graham and J. R. Walton, Springer-Verlag, Vienna, 1995

[21] M. Fabrizio, C. Giorgi, and A. Morro, Internal dissipation, relaxation property and free energy in materials with fading memory, J. Elasticity 40, 107-122 (1995)

[22] A. Morro, Wave solutions in linear viscoelastic materials, in Crack and Contact Problems for Viscoelastic Bodies, eds. G. A. C. Graham and J. R. Walton, Springer-Verlag, Vienna, 1995

[23] G. Del Piero and L. Deseri, On the concepts of state and free energy in linear viscoelasticity, Arch. Rational Mech. Anal. 138, 1-35 (1997)

[24] G. Del Piero and L. Deseri, On the analytic expression of the free energy in linear viscoelasticity, J. Elasticity 43, 247-278 (1996)

[25] M. E. Gurtin and W. J. Hrusa, On energies for nonlinear viscoelastic materials of single-integral type, Quart. Appl. Math. 46, 381-392 (1988)

[26] J. M. Golden and G. A. C. Graham, Boundary Value Problems in Linear Viscoelasticity, SpringerVerlag, Berlin, 1988

[27] N. I. Muskhelishvili, Singular Integral Equations, Noordhoff, Groningen, 1953

[28] M. E. Gurtin and I. Herrera, On dissipation inequalities and linear viscoelasticity, Quart. Appl. Math. 23, 235-245 (1965)

[29] E. C. Titchmarsh, Introduction to the Theory of Fourier Integrals, Clarendon, Oxford, 1937

[30] I. N. Sneddon, The Use of Integral Transforms, McGraw-Hill, New York, 1972

[31] P. M. Morse and H. Feshbach, Methods of Theoretical Physics, McGraw-Hill, New York, 1953 